\title{
An influence of shroud design parameters on the static stresses of blade assemblies
}

\author{
Kyrylo Savchenko ${ }^{1, *}$, Anatoliy Zinkovskii ${ }^{1}$, Romuald Rzadkowski ${ }^{2}$, Radoslaw Przysowa ${ }^{3}$, \\ and Vadym Kruts ${ }^{1}$ \\ ${ }^{1}$ IPS NASU, Department of Oscillations and Vibration Reliability, 01014 Kyiv, Ukraine \\ ${ }^{2}$ IMP PAN, Aeroelasticity Department, 80-231 Gdansk, Poland \\ ${ }^{3}$ ITWL, Aeroengine Department, 01-494 Warsaw, Poland
}

\begin{abstract}
In this study, the structural analysis of the blade assemblies was carried out using the finite element method to determine the influence of design parameters of shroud couplings on the static stresses of turbine rotor blades with zigzag and slant shroud couplings. An angle of inclination of the shroud contact surfaces with respect to the rotor rotation axis was selected as the design parameter. Based on the calculation results, it has been found that irrespective of the type of the shroud coupling, the values of the contact pressure and the stresses in the shroud increase with the angle of inclination of the contact surfaces. Also, for the slant shroud coupling, the stresses increase in the blade airfoil portion with the increase of angle of inclination of the contact surfaces, while for the zigzag shroud coupling the contact stresses decrease with the increase of this angle. It was concluded that the zigzag shroud coupling causes the increase in static stresses when compared to the slant one.
\end{abstract}

\section{Introduction}

Rotating blades are highly stressed components of turbomachinery. In the practice of modern aircraft engine design, estimation and reduction of blade vibration level is an important task to decrease the risk of blade failure during the engine operation. To reduce the vibration level, material or fluid damping are insufficient in most cases, therefore friction damping at different blade joints has to be taken into account. Different friction damping methods can be used to decrease blade vibration, for example, shroud coupling [1-4], under platform dampers $[5,6]$, or blade-disk joints $[1,7]$. Moreover, for some tasks, the damping coatings for blade airfoil can be considered [8]. Such methods of energy dissipation allow for decreasing of blade assemblies' sensitivity to vibration excitation.

In turbomachinery design, two types of shroud coupling, namely zigzag and slant shrouds, are used to increase the serviceability of turbine rotor blades. Advantages and disadvantages of these methods were discussed in [9] where the recommendations concerning the application areas were also given.

\footnotetext{
* Corresponding author: savchenko@ipp.kiev.ua
} 
Considerable attention of researchers has been paid to the study of the influence of the design process as well as operating factors on the stresses of shrouded turbine rotor blades as a pre-requisite of their vibration strength analysis, as evidenced by the investigation results [2, 10-12]. Szwedowicz et al. [2] present the results of the influence of the contact orientation angle between shrouds on the contact force and contact area as well as free vibration of the blades with different shroud configurations. However, only the beam blade model was considered in this investigation. Krivoshei [10] use an analytical model to determine the influence of the inclination of the contact surfaces on the contact pressure along the shrouds. Fan et al. [11] propose optimization of the zigzag shroud using a package of three blades, which leads to the stiffening of the coupling and may generate incorrect results. In [12] authors study the influence of the inclination angle of the contact surfaces and operation factors on the static stresses of the blades with slant shrouds. The aim of this current work, which is a continuation of [12], is analysis of the influence of the shroud coupling characteristic, such as the orientation of the shroud contact surfaces, defined by the angle of their inclination relative to the disk rotation plane on the static stresses of the blades with different shroud configurations. Results of this research can help to estimate blade lifetime at the stage of turbomachinery development.

\section{Object of investigation and its simulation}

Two assemblies of a high-bypass turbofan engine, whose blades are characterized by zigzag and slant shrouds, were chosen as the objects of investigation for computational experiments. They contain 136 blades $(S=136)$ made of heat-resistant alloy ZhS26-VI. Fragments of the assemblies under investigation are presented in Fig. 1.

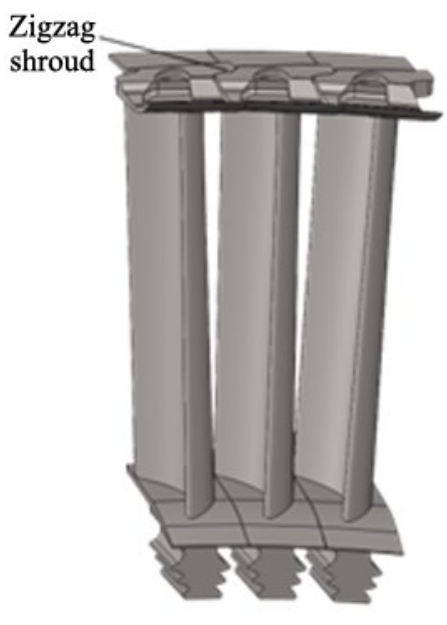

$a$

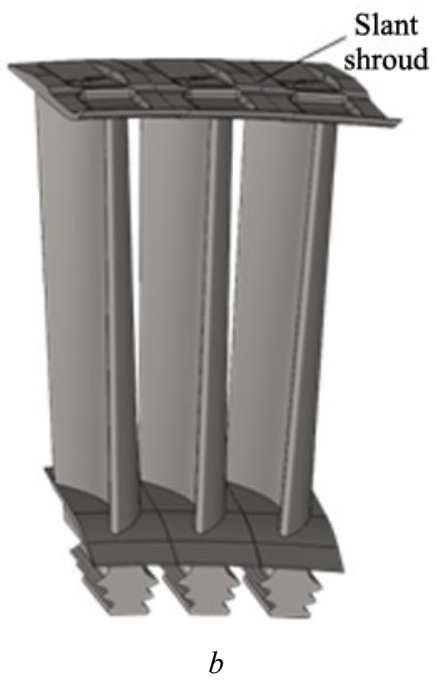

Fig. 1. Fragments of the blade assemblies with zigzag $(a)$ and slant $(b)$ shroud coupling.

The shrouds of the adjacent blades of the assembly interact along the contact surfaces $K$, as it is shown in Fig. 2. Here $\alpha$ is the inclination angle of the blade shrouds surfaces $K$ with respect to the plane of rotation of the disk, $t_{s}$ is the spacing between blades of the blade assembly, and $F$ is the resultant of the normal forces acting on the surfaces $K$. 


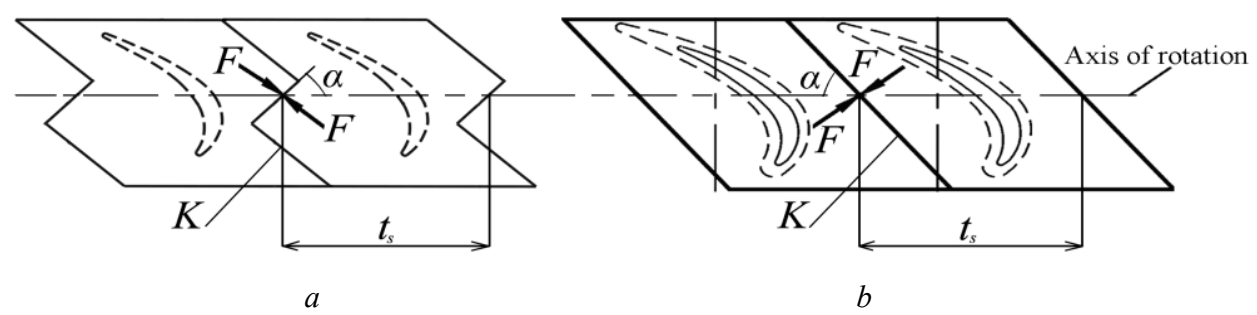

Fig. 2. Schematic of the interaction of zigzag $(a)$ and slant $(b)$ shroud coupling.

In the study we employed a finite element method (FEM) as one of the most developed computer simulation methods, which allows to reduce the number of expensive bench tests.

For ANSYS FE modelling of the shrouded blades under investigation with allowance for their design features, we divided the airfoil into portions, which were characterized by a small change in the twist angle. In that case, a linear eight-node finite element SOLID45 and its modifications were chosen for modelling. Typically, course elements were used at the blade zones without abrupt variations in the strains or stresses distribution, mainly in the blade airfoils, while fine elements were used for the mesh close to the stress concentration areas and in the shroud coupling zone. It should be noted that the calculation results accuracy may decrease if the dimensions of the neighbouring elements located close to the stress concentration area differ significantly. Therefore, to obtain more accurate results, we did not use narrow and elongated finite elements. A general view of the blade FE model is shown in Fig. $3 a$.

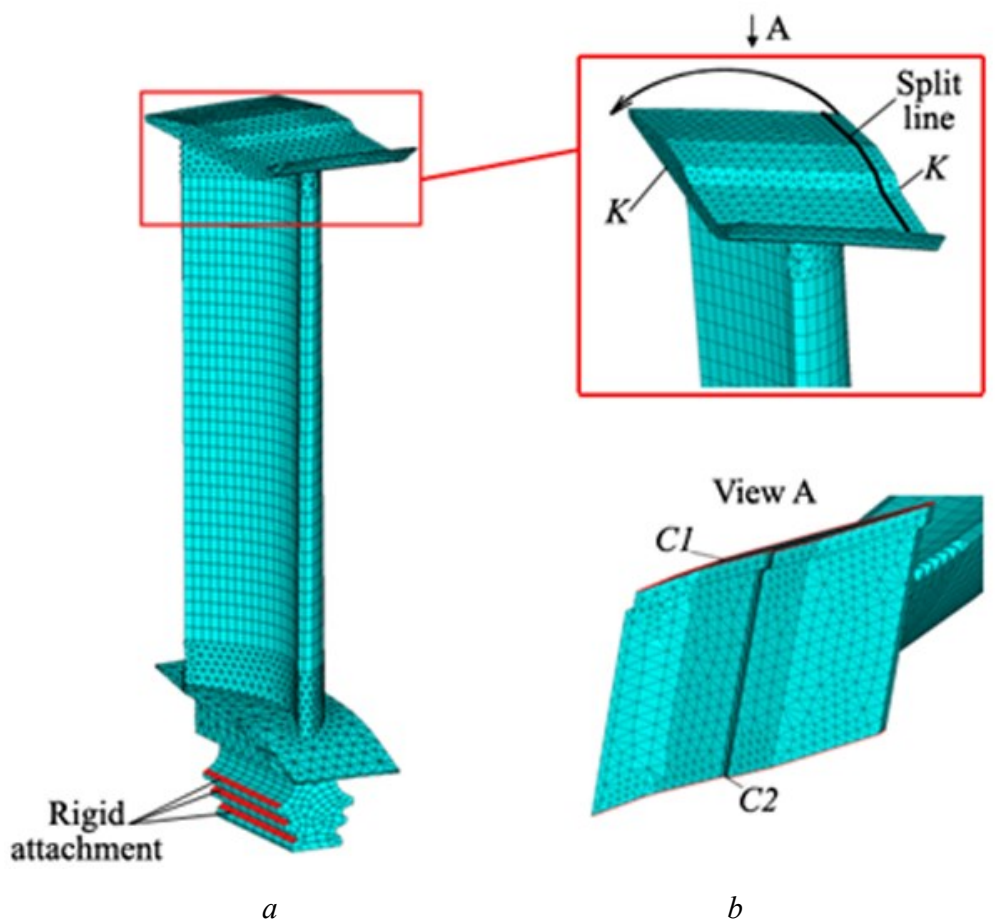

Fig. 3. The FE model of the shrouded blade $(a)$ and schematic of the contact interaction modelling $(b)$

Typical blade assemblies are characterized by cyclic symmetry. In this case, the problem solution is limited to the consideration of the individual sector with the appropriate boundary 
conditions. As a rule, the sector with one blade was used, and the cyclic symmetry order coincided with the number of blades.

To satisfy the boundary conditions, the shroud was cut so that its parts were connected along the common surface, whereas the nodes on this surface coincided. Then the cutoff part of the shroud with the FE mesh was transferred to the cylindrical system of coordinates through the angle corresponding to the period (Fig. $3 b$ ). In this way, tension was simulated along the contact surfaces $K$ and conjugation boundary conditions were specified on the cut surfaces $C 1$ and $C 2$.

To ensure the accurate simulation of the interaction of blades over the contact surfaces of shrouds, the surface-surface contact elements, CONTA175 and TARGE170, were used, which makes it possible to take into account different contact conditions of shrouds, such a tension, clearance, coupling, slippage, friction, or shear, which occur during operation.

To rule out the influence of the lock joint on the research results, it was assumed that there is a rigid coupling over the contact surfaces of the root of blades and the disk.

\section{Results of the computational experiments}

A comprehensive set of computational experiments has been performed involving the determination of the influence of the inclination angle $\alpha$ (in the range of $45^{\circ}$ to $65^{\circ}$ ) on the static stresses characteristics, for which the contact pressure $P_{\max }$ along the shrouds and the stresses $\sigma_{i}$ in the shroud and blade airfoil were selected.

In operation, the centrifugal force generated by the disk rotation and high temperatures of the gas flow act on the turbine rotor blades. Therefore, to solve the problem, the following parameters were chosen: the disk rotation frequency $\omega$ equal to 3790,5180 and $5850 \mathrm{rpm}$, and the temperature of gases behind the turbine $T$ of $905^{\circ} \mathrm{C}$. These parameters are typical for the engine analyzed [12].

Initially, to determine the influence of these two factors on the stresses of the rotor blades under study, the computational experiments were carried out in the absence of the centrifugal force field, i.e. for $\omega=0$ and at temperature $T=20^{\circ} \mathrm{C}$. The following values of the physical and mechanical characteristics of the material were used: elastic modulus of the 1st kind $E=$ $1.9 \times 10^{11} \mathrm{~Pa}$, density $\rho=8570 \mathrm{~kg} / \mathrm{m}^{3}$, and Poisson's ratio $\mu=0.3$.

Based on the calculations results, the diagrams of the maximum values of the selected characteristics vs. the value of the inclination angle $\alpha$ are plotted and compared in Fig. 4.

The analysis of the obtained distributions stresses has shown the following:

(i) Irrespective of the shroud coupling type, the values of the contact pressure and the stresses in the shroud increase with the inclination angle of the contact surfaces. For the slant shroud coupling, the stresses increase in the blade airfoil with the increase of inclination angle of the contact surfaces, while for the zigzag coupling they decrease. Also, the zigzag shroud coupling causes the increase in the static stresses in comparison to the slant coupling.

(ii) With the increase in the rotational speed, the increase in the maximum values of the contact pressure and the stresses in the blade shroud and the blade airfoil occurs in the whole range of the inclination angle of the contact surfaces.

For the temperature $T=905^{\circ} \mathrm{C}$, the computations were carried out using the assumption of the uniform distribution of temperature along the blade length. The modulus of elasticity of the material under these conditions was assumed $E_{T}=1.05 \times 10^{11} \mathrm{~Pa}$.

Based on the obtained results of calculations, the diagrams were plotted showing the variation of the maximum values of static stresses in the blade with the inclination angle of the contact surfaces for different values of the rotational speed, for temperature of $905^{\circ} \mathrm{C}$ as illustrated in Fig. 5. 


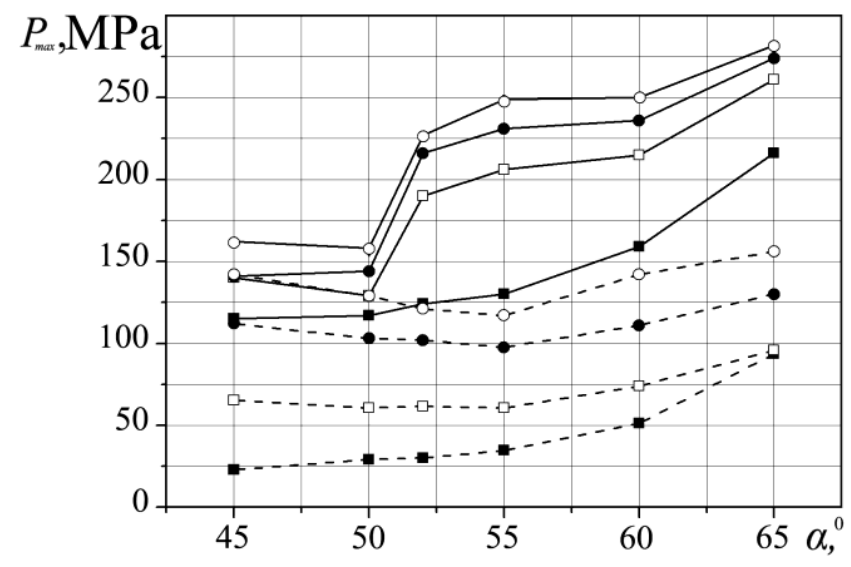

$a$

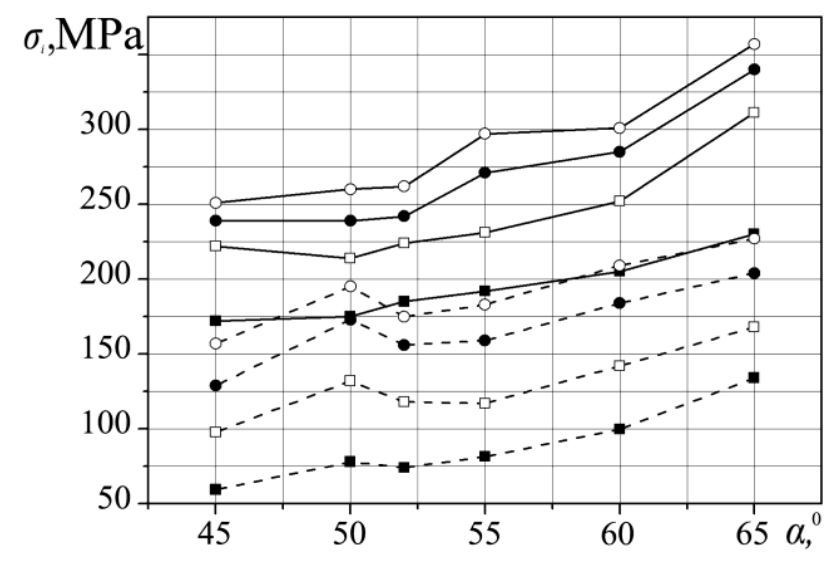

$b$

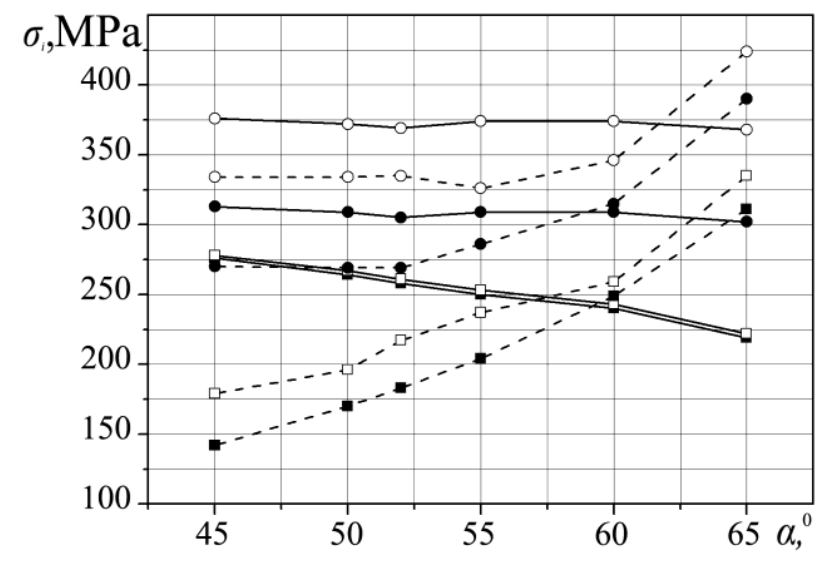

$c$

Fig. 4. Maximum contact pressure (a) and the stress in the shroud $(b)$ and in the airfoil $(c)$ of the blade as a function of the inclination angle of the contact surfaces relative to the rotation plane of the disk with the zigzag (solid lines) and slant (dashed lines) shroud coupling at $T=20^{\circ} \mathrm{C}$ and $\omega=0(\mathbf{\square}), 3790$ (), $5180(\bullet)$, and $5850 \mathrm{rpm}(\mathrm{\circ})$ 


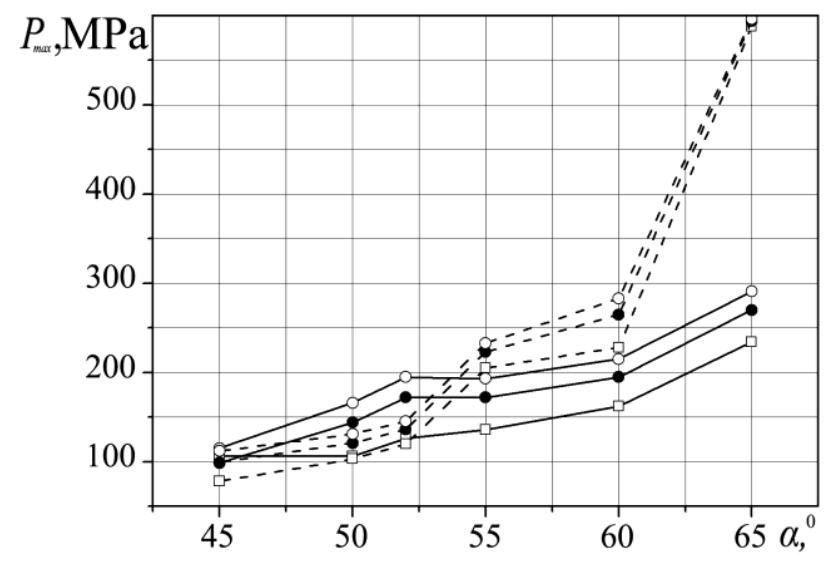

a

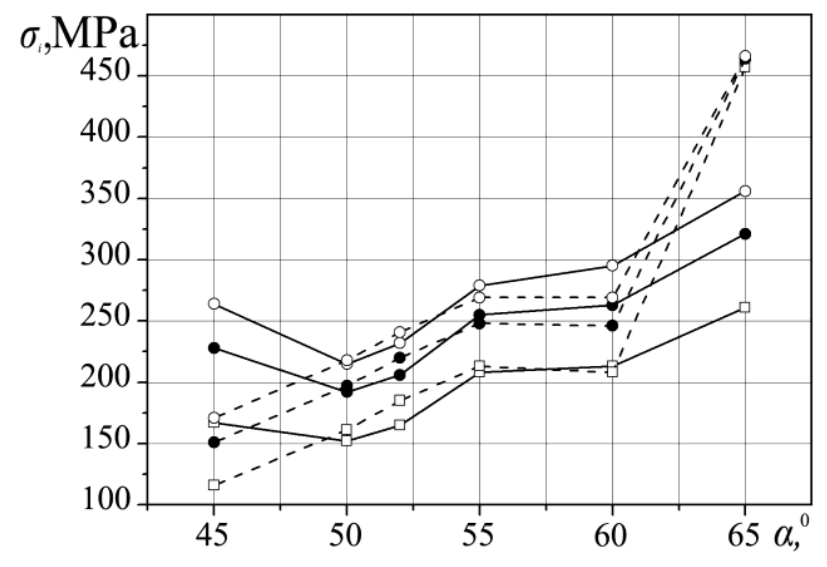

$b$

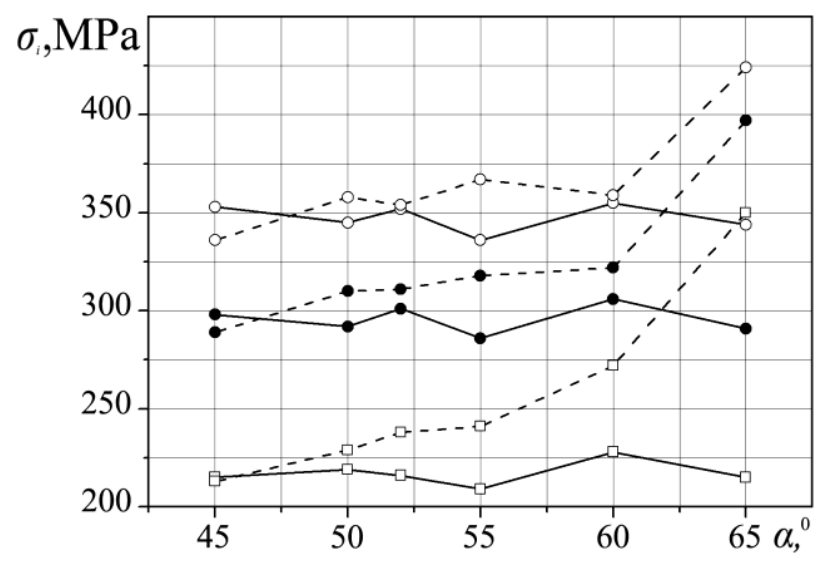

$c$

Fig. 5. Maximum contact pressure (a) and the stress in the shroud (b) and in the airfoil (c) of the blade as a function of the inclination angle of the contact surfaces relative to the rotation plane of the disk with the zigzag (solid lines) and slant (dashed lines) shroud coupling at $T=905^{\circ} \mathrm{C}$ and $\omega=3790$ (口), $5180(\bullet)$, and $5850 \mathrm{rpm}(\mathrm{\circ})$ 
Increasing temperature increases the values of the stresses in the blades with the slant shroud coupling and slightly decreases them for blades with the zigzag coupling in comparison to the results at $T=20^{\circ} \mathrm{C}$. This can probably be explained by thermal expansion of the material, which is responsible for the change in blades geometry. Moreover, for $\alpha=$ $65^{\circ}$, an abrupt increase of the contact pressure and the stress in the slant shroud was observed. It indicates that a further increase in $\alpha$ may adversely affect the lifetime of the blades.

The comparison between the obtained results of the simulations and the data in $[2,10]$ has shown good qualitative agreement (Fig. 6).

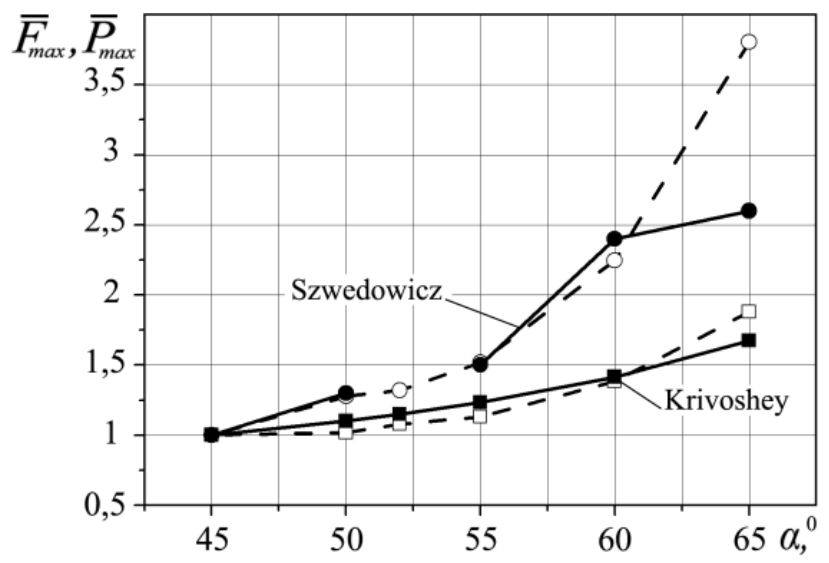

Fig. 6. Maximum relative normal forces (solid lines) and contact pressure (dashed lines) in the shroud as a function of the inclination angle of the contact surfaces relative to the rotation plane of the disk with the zigzag $(\boldsymbol{\bullet}, \square)$ and slant $(\bullet, \circ)$ shroud coupling

\section{Conclusions}

Based on the computational experiments performed and their results comparison with the known data, it was found that:

1. Irrespective of the type of the shroud coupling and the effect of centrifugal force and high temperature, the values of the contact pressure and the stress in the shroud increase in the whole range of variation of the inclination angle of the contact surfaces. In the blade airfoil with the slant shroud coupling, the values of the stress increase, whereas in that with the zigzag coupling, they decrease with an increasing inclination angle of their contact surfaces.

2. On the one hand, a consideration of the centrifugal force field results in the increase in the stresses in the blades in the whole range of variation in the inclination angle of the contact surfaces. On the other hand, consideration of the temperature increases the values of the studied stresses in the blades with the slant shroud coupling, and slightly decreases them for blades with the zigzag coupling.

3. Generally, the zigzag shroud coupling is responsible for the increase in the static stress in blades as compared to that in the blades with the slant shroud coupling.

4. The comparison between the obtained results of the computational investigations and the known data has shown good qualitative agreement. It indicates adequacy of the FE models developed and the conclusions drawn.

5. The method presented and the results obtained can be used for optimization of the existing high-bypass turbofan engines, as well as for analysis of dynamic behaviour of shrouded blade assemblies. 
This publication was prepared within the framework of the AERO-UA project, which has received funding from the European Union's Horizon 2020 research and innovation programme under grant agreement No 724034 .

We gratefully acknowledge Dr Wieslaw Beres from National Research Council Canada for his valuable suggestions and discussions.

\section{References}

1. I.G. Tokar, A.P. Zinkovskii, V.V. Matveev, Strength Mater., 35, 368-375 (2003)

2. J. Szwedowicz, R. Visser, W. Sextro, P.A. Masserey, J. of Turbomachinery, 130, 11-18 (2008)

3. K. Savchenko, A. Zinkovskii, I. Tokar, Proc. of the 25th Int. Congress on Sound and Vibration, 1-6 (2018)

4. R. Rzadkowski, Dynamics of Rotor Steam Turbine Blading. Part Two, Bladed Discs (Ossolineum, Wroclaw, 1998)

5. K.Y. Sanliturk, D.J. Ewins, A.B. Stanbridge, J. of Eng. for Gas Turbines and Power, 123, 919-929 (2001)

6. C.M. Firrone, S. Zucca, M.M. Gola, Int. J. of Non-Linear Mech., 46, 363-375 (2011)

7. E.P. Petrov, D.J. Ewins, J. of Turbomachinery, 128, 403-410 (2006)

8. A.I. Ustinov, A.P. Zinkovskii, I.G. Tokar, V.S. Skorodzievskii, Advances of Electrometallurgy, 8, 31-38 (2010)

9. V.M. Merkulov, F.D. Ilyushchenko, Vestn. Dvigatelestr., 2, 114-117 (2005)

10. V.Ya. Krivoshei, Strength Mater., 14, 1062-1064 (1982)

11. J. Fan, L. Han, R. Wang, X. Shen, W. Zeng, Z. Chang, Proc. of ASME Gas Turbine India Conference, 657-663 (2012)

12. K.V. Savchenko, A.P. Zinkovskii, I.G. Tokar, Ya.D. Kruglii, Strength Mater., 46, 493502 (2014) 